\title{
Article \\ Hubble Expansion as an Einstein Curvature
}

\author{
John Herbert Marr ${ }^{1}$ \\ 1 Unit of Computational Science, Building 250, Babraham Research Campus, Cambridge, CB22 3AT, UK; \\ john.marr@2from.com
}

\begin{abstract}
Hubble expansion may be considered as a velocity per photon travel time rather than as velocity or redshift per distance. Dimensionally, this is an acceleration and will have an associated curvature of space under general relativity. This paper explores this theoretical curvature as an extension to the spacetime manifold of general relativity, generating a modified solution with three additional non-zero Christoffel symbols, and a reformulated Ricci tensor and curvature. The observational consequences of this reformulation were compared with the $\Lambda \mathrm{CDM}$ model for luminosity distance using the extensive type Ia supernovae (SNe Ia) data with redshift corrected to the $\mathrm{CMB}$, and for angular diameter distance with the recent baryonic acoustic oscillation (BAO) data. For the SNe Ia data, the modified GR and $\Lambda$ CDM models differed by ${ }_{-0.15}^{+0.11} \mu_{B}$ mag. over $z_{c m b}=0.01-1.3$, with overall weighted RMS errors of $\pm 0.136 \mu_{B}$ mag for modified GR and \pm 0.151 $\mu_{B}$ mag for $\Lambda$ CDM espectively. The BAO measures spanned a range $z=0.106-2.36$, with weighted RMS errors of $\pm 0.034 \mathrm{Mpc}$ with $H_{0}=67.6 \pm 0.25$ for the modified GR model, and $\pm 0.085 \mathrm{Mpc}$ with $H_{0}=70.0 \pm 0.25$ for the $\Lambda \mathrm{CDM}$ model. The derived GR metric for this new solution describes both the $\mathrm{SNe}$ Ia and the BAO observations with comparable accuracy to $\Lambda \mathrm{CDM}$ without requiring the inclusion of dark matter or $w^{\prime}$-corrected dark energy.
\end{abstract}

Keywords: cosmology, theory; cosmology, observations; Hubble flow; dark energy; dark matter 


\section{Introduction}

To the early successes of the precession of the perihelion of Mercury and gravitational bending of star light during a solar eclipse have been added many other observations confirming that the behaviour of masses and photons in a local gravitational field is well described by General Relativity (GR). Oservational data have confirmed without exception that solutions to the general field equations are exact when applied to static or rotating localised gravitational masses, including gravitational redshift [1], the production of Einstein rings by DM halos [2], X-ray emission data in the neighbourhood of black holes [3-5], and the Sunyaev-Zeldovich effect [6,7].

The standard definition of the Hubble expansion coefficient is a velocity per distance $(\mathrm{km} / \mathrm{s} / \mathrm{Mpc})$, but can also be thought of as a velocity per photon travel time, which is dimensionally an acceleration. This pseudo-acceleration arises directly from the first postulate of special relativity (SR) that the velocity of light $c$ is constant for all observers in their local reference frame. Taking $H_{0}=67.7 \mathrm{~km} / \mathrm{s} / \mathrm{Mpc}$ gives an equivalent value of $H_{0} \equiv 20.74 \mathrm{~km} / \mathrm{s} / \mathrm{Myr}$ for photon travel time across the Hubble expansion. By considering this as an acceleration, an additional curvature of space is generated requiring an extension to Einstein's general equation and producing a solution with three additional non-zero Christoffel symbols and a reformulated Ricci tensor and curvature. This solution to Einstein's equation retains the standard components of GR, while reducing to the equations of SR as $\Omega_{m} \rightarrow 0$ without requiring a change in $\Omega_{k}$, the spatial curvature term. Sections 6 and 7 examined the model by comparing its predictions for luminosity distance with the extensive apparent magnitude data of supernovae type 1a (SNe 1a) and a wide range of recently published angular diameter distances out to $z=2.36$. On both measures, it was comparable to the best $w^{\prime} \mathrm{CDM}$ models [8].

\section{The FLRW metric}

Geometrically, the constancy of $c$ for any observer may be represented by the locus of a logarithmic spiral to generate a curve of constant angle to the local time axes [9] (Fig. 1). This geodesic of SR may be illustrated as a hyperbolic curve crossing diverging imaginary time axes, and is independent of the spatial curvature which is allowed to be flat, spherical or hyperbolic. The Friedmann, Lemaitre, Robertson, Walker (FLRW) equation allows this expansion curvature of SR to be introduced by the hyperbolic curvature of space as the combined mass-energy of space $\rightarrow 0$, which contrasts with observations that show space to be essentially flat. In GR, curvature occurs by the distortion of space by gravitational energy, and these gravitational effects on the curvature of the Universe will increase in significance as look-back time extends and temperature and energy densities increase towards the CMB radiation and the early universe at $z \simeq 1090$ [7]. The loss of an innate hyperbolic curvature of expansion may be mimicked in GR by introducing extra mass as dark matter (DM) and dark energy as a variable acceleration component, with both components being required and adjusted to match current cosmological observations.

A model geometry of the evolving Universe may be constructed as a simply connected smooth Riemannian manifold $R_{m}$ with metric $g_{\mu v}$. It is taken as axiomatic that the Universe is homogeneous and isotropic in space, but not in time. Of the eight Thurston 3-manifold Riemannian geometries, only three fulfil the criteria of homogeneity and isotropy for the observable Universe: the 3-sphere $S^{3}$, the 3-D Euclidean space $E^{3}$, and the 3-D hyperbolic space $H^{3}$. Finite volume manifolds with $E^{3}$ geometry are all compact, and have the structure of a Seifert fibre space, remaining invariant under Ricci flow. $S^{3}$ manifolds are exactly closed 3-manifolds with a finite fundamental group, and under Ricci flow such manifolds collapse to a point in finite time. In contrast, manifolds with $\mathrm{H}^{3}$ hyperbolic geometry are open and expand under Ricci flow [10]. Using a Lie group acting on the metric to compute the Ricci tensor $R_{\mu v}$, these manifolds are deformed by Ricci flow as a function of time $t$ and we may then define the geometric evolution equation, $\partial_{t} d_{i j}=-2 R_{i j}$, with normalised Ricci flow given by [11]: 


$$
\partial_{t} g_{i j}=-2 R_{i j}+\frac{2}{3} R g_{i j}
$$

This is equivalent to a Universe that can be foliated into space-like slices, and spacetime itself may therefore be represented by $\Gamma-\mathbb{R}^{3}$ where $\Gamma$ represents the time direction, with the general form $\mathrm{d} s^{2}=g_{\mu \nu} \mathrm{d} x^{\mu} \mathrm{d} x^{\nu}$ in the standard notation. $\mathbb{R}^{3}$ must be a maximally symmetric space to conform to a homogeneous and isotropic three-manifold, with metric $\mathrm{d} \sigma^{2}=\gamma_{i j} \mathrm{~d} x^{i} \mathrm{~d} x^{j}$. By scaling $t$ such that $g_{00}=-1$ with $\mathrm{c}=1$, we may write the metric as:

$$
\mathrm{d} s^{2}=-\mathrm{d} t^{2}+a(t)^{2} \gamma_{i j}(x) \mathrm{d} x^{i} \mathrm{~d} x^{j}
$$

where $\gamma_{i j}, x^{i}, x^{j}$ are the co-moving co-ordinates.

In cosmology, homogeneity and isotropy imply that $\mathbb{R}^{3}$ has the maximum number of Killing vectors, and with the additional constraint of the metric being torsion-free (the Levi-Civita connection), $\gamma_{i j}$ is the maximally symmetric metric of $\mathbb{R}^{3}$. This yields the general solution to Einstein's equation [12-14] which may be stated in polar coordinates (Eq. 3):

$$
\begin{gathered}
\mathrm{d} s^{2}=-\mathrm{d} t^{2}+a(t)^{2}\left[\mathrm{~d} r^{2}+S_{k}^{2}(r) \mathrm{d} \Omega^{2}\right], \\
\text { where } S_{k}^{2}(r) \equiv \begin{cases}\Re_{0}^{2} \sin ^{2}\left(r / \Re_{0}\right) & \text { for } \Re_{0}>0 \\
r^{2} & \text { for } \Re_{0}=\infty \\
\Re_{0}^{2} \sinh ^{2}\left(r / \Re_{0}\right) & \text { for } \Re_{0}<0,\end{cases} \\
\text { or } S_{k}(r) \equiv \frac{1}{\sqrt{ } K} \sin (r \sqrt{K}),
\end{gathered}
$$

and $K=\operatorname{sgn}\left(\Re_{0}\right) / \Re_{0}^{2}$ is the curvature. With $\chi$ as a third angular coordinate, $r=\Re_{0} \chi$ is the radial distance along the surface of the manifold, $\Re_{0}$ is the comoving 4-space radius of $\mathbb{R}^{3}$ at the present epoch, and $\mathrm{d} \Omega^{2}=\mathrm{d} \theta^{2}+\sin ^{2} \theta \mathrm{d} \phi^{2}$ is the angular separation. The signature $\operatorname{diag}(-,+,+,+)$ defines this as a Pseudo-Riemannian manifold with metric $g_{\mu v}$ and spatial metric $g_{i j}$, and $a(t)$ is the scale factor at proper time $t$. The actual form of $a(t)$ is determined by the curvature of the manifold and the energy tensor of Einstein's field equations, with curvature $K$ (or radius $\Re$ ), and scale factor $a(t)$ to be determined. The curvature or shape of the homogeneous hyper-surfaces are defined by the spatial 3-metric $\gamma_{i j} \mathrm{~d} x^{i} \mathrm{~d} x^{j}$ of Eq. (2), but the whole dynamics of the Universe are embodied only in the expansion factor, $a(t)$ [12].

Just as the surface of a sphere is a curved 2-D manifold embedded in Euclidean 3-space, this manifold is a curved 3-D volume embedded in Euclidean 4-space. Measurements on the surface of a 2-D sphere involve a distance and an angle, with the third dimension the implicit radius of the sphere. For the 3-D volume, $\chi$ is a third angular measure, with the implicit radius $\Re$ now the fourth dimension [15]. For an expanding 2-D manifold in 3-D space, time is geometrically a fourth dimension, and-by extension-for the expanding 3-D volume in 4-D space, time must be represented geometrically as a fifth dimension. To understand physical reality we may invoke geometrical representations, with intrinsic curvature equivalent to embedding in higher dimensions. However it must be emphasised that intrinsic curvature is a mathematical construct relating the deviation of parallel lines towards or away from each other and does not require higher dimensions. This purely geometric dimensionality is distinct from other attempts to introduce extra physical dimensions into GR by quantum gravity or string or loop theory [16].

With $r$ as the radial coordinate, radial distances are Euclidean but angular distances are not. If we are only interested in photon redshift distances, $\mathrm{d} \Omega=0$ and Eq. (3) is the more useful form of the metric. Setting $\mathrm{d} s^{2}=0$ and $g_{\theta \theta}=g_{\phi \phi}=0, \mathrm{~d} r$ now represents a radial photon distance from the era of emission $t_{e}$ to the present epoch at $t_{0}$, with: 


$$
R_{\gamma}=\int \mathrm{d} r=\int_{t_{0}}^{t} \frac{\mathrm{d} t}{a(t)} .
$$

$R_{\gamma}$ is a function of $a(t)$ only, and may be independent of the curvature of the spatial manifold. Symmetry ensures that proper time for standard clocks at rest relative to the spatial grid is the same rate as the cosmological time $(t)$, making the interval $\mathrm{d} t$ Lorentzian. Any coordinate system in which the line element has this form is said to be synchronous because the coordinate time $t$ measures proper time along the lines of constant $x^{i}$ [12].

The substitution $\chi=\sin (r / \Re), \chi=r / \Re$, or $\chi=\sinh (r / \Re)$ into $S_{k}(r)$ in Eq. (3) makes $\chi$ a radial coordinate with $\Re$ absorbed into $a(t)$, and now angular distances are Euclidean but radial distances are not (Eq. 7):

$$
\mathrm{d} s^{2}=-\mathrm{d} t^{2}+\Re(t)^{2}\left[\frac{\mathrm{d} \chi^{2}}{1-k \chi^{2}}+\chi^{2} \mathrm{~d} \Omega^{2}\right]
$$

This form is useful for measuring angular distances on a shell of fixed radius $\left(g_{\chi \chi}=(1-\right.$ $\left.k \chi^{2}\right)^{-1}, \mathrm{~d} \chi=0$ ), such as the proper diameters of clusters or spatial volume for galaxy counts.

\section{The expanding Universe as geometry}

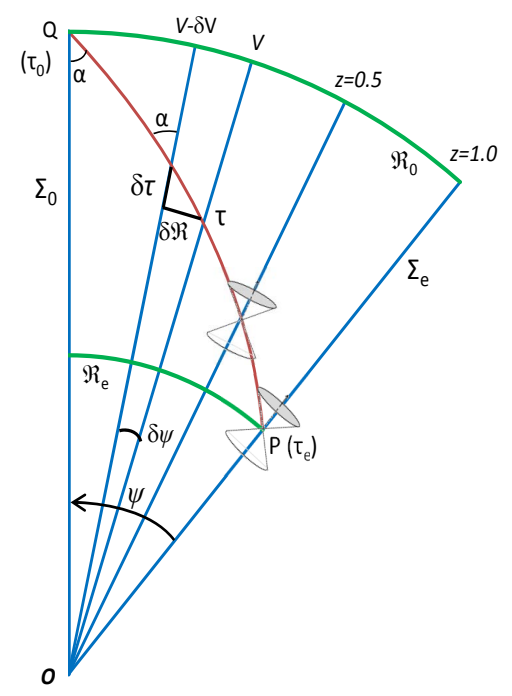

Figure 1. Geodesic for a photon traversing mass-free space, from frame $\Sigma_{e}$ moving at velocity $V$ relative to an observer $\Sigma_{0}$, with a small element of the geodesic $\delta S$ for reference frames $V$ and $V-\delta V$ rotated through $\delta \psi$, with two local light cones. The photon path (red line) is a logarithmic spiral, $1+z=\exp \psi$, across diverging galaxies on the complex plane $\left(c=1 \equiv 45^{\circ}\right)$ [9]. Redshifts referenced to $\Sigma_{0}$.

Milne described a dust universe expanding with constant relative velocity assigned to each galaxy, and with a mass-energy density sufficiently low that any deceleration could be neglected [17]. Such a universe does not have to be spatially flat, but it does have the property that $\dot{a}(t)=$ constant, and hence $a(t)=a_{0}\left(t / t_{0}\right)$, where $a_{0}$ is the scale factor at the current epoch $t_{0}$, defined to be $a_{0}=1$. Taking Eq. (3) to be the FLRW metric for the photon path, we may state that $\mathrm{d} \theta=\mathrm{d} \phi=0$, and hence $\mathrm{d} \Omega=0$ and consider only the radial coordinate $\mathrm{d} r$. This modified Milne model (M3) is therefore independent of the space curvature: this may be an expanding 3-sphere, a flat 3-sheet, or a 3-saddle. What M3 does demand is that the time-like foliation of these 3-spaces is linear; the space itself may 
be infinite or closed, but will maintain its initial curvature signature whether expanding forever or contracting.

Einstein's first postulate in a system of non-accelerating inertial frames may be summarised as: the velocity of light is constant for any observer, independent of the velocity of the source. Interpreting the time coordinate as the imaginary axis has become depreciated, but to do so forces the proper time axis to be a radius of length $\tau=i c t$ and allows a graphical interpretation of the interval $S$ to be unvarying under rotation, providing a geometric visualisation to this postulate [9]. In Figure 1, the infinitesimal geodesic is extended to illustrate the path of photons between galaxies in the uniformly expanding homogeneous, isotropic universe of M3.

This geometrical figure is generated by assuming that: (1) observed redshifts represent a true relative motion (whatever the underlying cause); (2) galaxies are moving apart with a velocity that is constant over the time period of the observations, generating a set of diverging inertial reference frames in space; (3) photons traverse these reference frames at constant velocity $c$ to all local observers, in their local Minkowski space under a Lorentzian transformation; (4) this is a 'dust' Universe, with no gravitational effects.

Any individual volume of space such as a specific galaxy may be considered stationary within its own reference frame. Let us define this reference frame as $\Sigma_{0}$ for our own local galactic space (Fig. 1). This neglects small-scale local movements, being a simple representation and first order approximation of an idealised world line for a particle in space, because the components of $v$ are assumed to relate only to local motions that are generally much less than the recessional velocity, and are taken to be zero in most theoretical models of the Universe.

The relative motion of two inertial frames, $\Sigma_{0}$ and $\Sigma_{e}$, diverging from a common origin with velocity $v$ may then be viewed as a hyperbolic rotation $\psi$ (the rapidity) of the spacetime coordinates on the imaginary plane (Fig. 1). This is a Lorentz boost with a rotational 4-matrix $\Lambda_{v^{\prime}}^{\mu}$ :

$$
\begin{gathered}
x^{\mu}=\Lambda_{v^{\prime}}^{\mu} x^{v^{\prime}} \\
\Lambda_{v^{\prime}}^{\mu}=\left(\begin{array}{cccc}
\cosh \psi & \sinh \psi & 0 & 0 \\
\sinh \psi & \cosh \psi & 0 & 0 \\
0 & 0 & 1 & 0 \\
0 & 0 & 0 & 1
\end{array}\right)
\end{gathered}
$$

where $\cosh \psi=\left(1-v^{2} / c^{2}\right)^{-1 / 2}=\gamma, \tanh \psi=v / c=\beta$, and $\sinh \psi=\beta \gamma$, in the standard notation, with $\operatorname{det} \Lambda=+1$.

Now consider a volume of space receding from us with velocity $v$ as defined by its redshift, with a proper radial distance $\Re_{e}$ at the time of emission. The photon path can now be represented geometrically as a logarithmic spiral on the complex plane ( $P Q$ in Fig. 1). It will be noted that $\psi$ is the hyperbolic angle, so the geometry allows $\psi>360^{\circ}$ because $v / c=\tanh \psi \rightarrow 1$ as $v \rightarrow c$ and $\psi \rightarrow \infty$, whereas local velocities are represented by real angles with trigonometric functions. The scale is chosen by convention such that $\alpha=45^{\circ}$ with $c=1$, hence the maximum angle in the local frame of reference corresponds to the standard light cone with $\operatorname{atan}(1)=45^{\circ}$. Although the spatial component of the M3 model may have curvature, M3 has no matter density and Fig. 1 is therefore geometrically flat as a consequence of the linear relationship between the radial and time axes.

For a photon, $\delta S=0$ (null geodesic for photon). It then follows that $\delta \Re^{2}=c^{2} \delta t^{2}$, or $\delta \Re= \pm c \delta t$, where the sign represents an incoming or outgoing photon. But $\delta \Re=c t \delta \psi$, thus $\delta t / t=\mp \delta \psi$. Using $-\delta \psi$ for the incoming photon and integrating:

$$
\begin{gathered}
\int_{t_{e}}^{t_{0}} \frac{\mathrm{d} t}{t}=\int_{\psi}^{0}-\mathrm{d} \psi . \\
\text { i.e. } \ln \left(t_{0} / t_{e}\right)=\psi \text { or } t_{0} / t_{e}=e^{\psi} .
\end{gathered}
$$


Although all diverging world lines are equivalent and will "see" photons intercepting and leaving them at velocity $c$, the source lines are Doppler red-shifted with a wavelength of emission $\lambda_{e}$ in $\Sigma_{e}$, and a wavelength at observation $\lambda_{0}$. Redshift is defined as:

$$
z=\frac{\lambda_{0}-\lambda_{e}}{\lambda_{e}}=\lambda_{0} / \lambda_{e}-1
$$

and setting $\lambda_{e}=\Delta t_{e}, \lambda_{0}=\Delta t_{0}$, it is easy to show that

$$
1+z=\Delta t_{0} / \Delta t_{e}=t_{0} / t_{e}=e^{\psi} .
$$

But $e^{\psi}=\cosh \psi+\sinh \psi$, hence

$$
1+z=\gamma+\gamma \beta=\gamma(1+\beta),
$$

which is the relativistic Doppler shift in SR, with $z \rightarrow \infty$ as $v \rightarrow c$. We may perform a topological transform of the Milne model of Fig. 1 into an imaginary 4-cone (Fig. 2) without loss of generality. From Eq. (12), $\psi=\log (1+z)$, and the three galaxies represented in Fig. 2, with redshifts of 0.5, 1.0 and 1.5 , have corresponding hyperbolic angles of $\psi=23.2^{\circ}, 39.7^{\circ}$, and $52.5^{\circ}$ respectively.

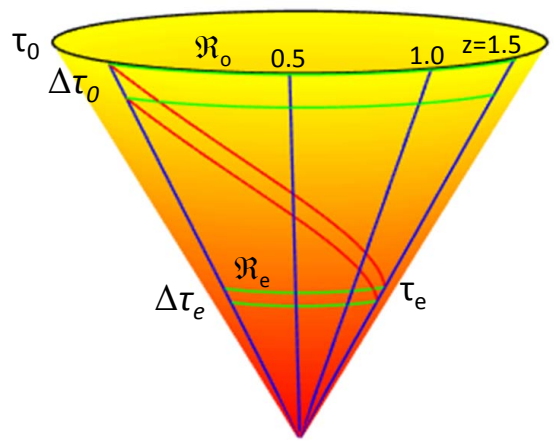

Figure 2. The Milne manifold of Fig. 1 as a 3-D cone for two photons crossing expanding space, originating at redshift $z=1.5$ and crossing the paths of galaxies at redshifts $z=1.0, z=0.5$, and $z=0$ at constant $\left(45^{\circ}\right)$ angles. The increase in Doppler wavelength $\left(\Delta \tau_{e}\right.$ to $\Delta \tau_{0}$ equivalent to $\lambda_{e}$ to $\left.\lambda_{0}\right)$ is visualised in this exaggerated plot.

Despite the appearance of curvature, there is no acceleration $(\dot{a}=$ constant; $\ddot{a}=0)$ and this remains a topologically flat figure. The imaginary proper time axes (e.g. $\tau_{0}$ and $\tau_{e}$ ) are straight lines that diverge linearly. Likewise, the radii of curvature round the vertical axis are proportional to $a(t)$, the radial distances on the manifold at constant cosmological (proper) times (e.g. $\Re_{0}$ and $\Re_{e}$ ) are orthogonal functions of $a(t)$ only, and the locus of each photon track is a line of constant angle.

\section{GR as geometry}

The presence of mass-energy in the Universe introduces a non-linear component to $a(t)$ with consequent curvature of the time axis, and an additional curvature to the path of the photon. This cannot be displayed on a flat 2-D diagram, but can be demonstrated using the topological transform of Fig. 2. The presence of acceleration now introduces curvature to the imaginary $\tau$ coordinate (Fig. 3), representing accelerations from gravitational or dark mass and dark energy that may be attractive/positive or negative/repulsive respectively.

The manifold of a sphere in 3-space is sufficiently described as a curved two-dimensional surface. Similarly the extra dimensions required to visualise the geometry of expanding curved spacetime do not represent real dimensions, but are a helpful aid to geometrical visualisation of the manifold. Because 3-space with curvature require a 4-dimensional space and the curved time 


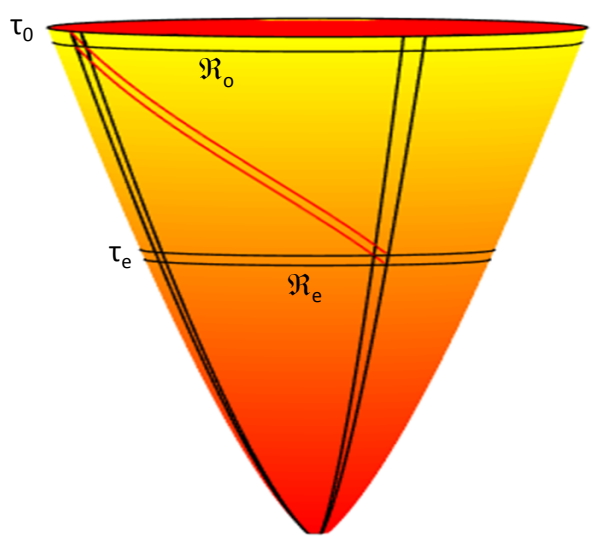

Figure 3. The cone manifold of Fig. 2 with curvature of the imaginary time axes by the presence of matter, and two photons crossing the expanding curved space at a constant $45^{\circ}$ angle.

coordinate occupies a further dimension, space-time now exists in 5-space, compacted in Fig. 3 to a 2-manifold in 3-space. Integration of the photon path across this surface may be represented by considering a thin wedge or petal of the time-space manifold in GR (Fig. 4), with the imaginary surface curved by mass-energy as well as by expansion.

The new radius of curvature is $R(\tau)=1 /(\mathrm{d} \beta / \mathrm{d} \tau)$, and this is independent of the spatial curvature, $K$. In the Milne model, the manifold is flat with $\mathrm{d} \beta / \mathrm{d} \tau=0$, and $R=\infty$, and the cone base angle, $\beta_{0}$, can take any arbitrary value, with $\beta_{0}=\pi / 2$ for Fig. 1 . Referring to Fig. 4 , the lines of longitude are the imaginary time axes, with $\mathrm{d} \tau=i \mathrm{~d} t$, whilst the lines of latitude represent the spatial component defined by $\mathrm{d} L=\gamma_{i j}(x) \mathrm{d} x^{i} \mathrm{~d} x^{j}$ (Eq. 2); $\Delta L_{0}$ is the comoving distance; $\Delta L=a(t) \Delta L_{0}$ is the proper distance at time $t$; and the curvature $1 / R^{2}=f(\ddot{a})$ is the acceleration. It may be noted that-in contrast to a standard radius $v$. time plot with $t$ as the vertical axis-the time axis is here embedded in the manifold. Unlike Fig. 2, the apex of this cone does not converge onto the vertical axis, but curls round itself as $R \rightarrow 0$ and $\ddot{a} \rightarrow \infty$. The model therefore still requires an inflationary scenario to close the gap and ensure causal connectedness.

\section{Geometry with curvature}

Geometrically, redshift is observed when otherwise parallel photon paths diverge from each other, as evidenced in the flat Minkowsky Milne model of Fig. 1. The modified GR model presents the geometrical curvature of diverging (redshifted) photons as a clear but separate curvature superimposed on both the secondary curvature of spacetime through gravitational mass and any intrinsic primary curvature of space itself.

Standard vectors are restricted in the presence of curvature on the spacetime manifold, but we may use Cartan vectors as operators [15]. Assign to each particle in the Universe the set of observer-dependent coordinates $x^{\mu}$. This represents an invariant line element with proper time $x^{0}=t$, whose spacetime geometry is encoded in the metric $\mathrm{d} s^{2}=g_{\mu \nu} \mathrm{d} x^{\mu} \mathrm{d} x^{v}$, with space coordinates $x^{i}=x^{i}(t)$. Free particles then move along curved geodesics, with 4-velocity

$$
U^{\mu}=\frac{\mathrm{d} x^{\mu}}{\mathrm{d} s} .
$$

With $t$ as a parameter, the spatial derivatives are the velocity components $U^{i}=\mathrm{d} x^{i} / \mathrm{d} t$, and we may introduce the differential operator $\mathrm{d} / \mathrm{d} t=U^{i} \partial / \partial x^{i}$, which is the directional derivative along the curve [15]. The components $U^{i}$ of the operator now form the local coordinate basis,

$$
\overrightarrow{\mathrm{U}}=\frac{\mathrm{d}}{\mathrm{d} t} ; \overrightarrow{e_{i}}=\frac{\partial}{\partial x^{i}}
$$




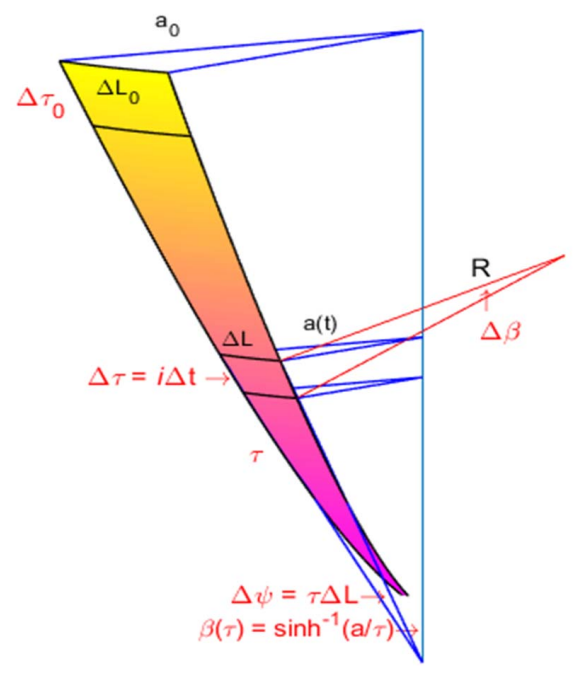

Figure 4. Thin slice of curved GR manifold, $\Delta L$ vs. $\tau$. Imaginary values are shown in red. $\Delta \beta=\Delta \tau / R$ is rate of change of expansion; $\beta=\sinh ^{-1}(a / \tau)=f(H)$. (The mass-energy radius of curvature, $R$, is considerably foreshortened in this exaggerated plot)

and the basis vectors $\vec{U}=U^{i} \overrightarrow{e_{i}}$ define the parameterised vector space associated with the point $x^{\mu}$.

Acceleration may be expressed in terms of Eq. (14):

$$
\frac{\mathrm{d} U^{\mu}}{\mathrm{d} s}=\frac{\partial U^{\mu}}{\partial x^{\alpha}} \frac{\mathrm{d} x^{\alpha}}{\mathrm{d} s}=U^{\alpha} \frac{\partial U^{\mu}}{\partial x^{\alpha}} .
$$

The motion is then described by the geodesic equation:

$$
\begin{gathered}
\frac{\mathrm{d} U^{\mu}}{\mathrm{d} s}+\Gamma_{\alpha \beta}^{\mu} U^{\alpha} U^{\beta}=0, \\
\text { i.e. } U^{\alpha}\left(\frac{\partial U^{\mu}}{\partial x^{\alpha}}+\Gamma_{\alpha \beta}^{\mu} U^{\beta}\right) \equiv U^{\alpha} \nabla_{\alpha} U^{\mu}=0,
\end{gathered}
$$

where $\Gamma_{\alpha \beta}^{\mu}$ are the Christoffel symbols, defined by:

$$
\Gamma_{\alpha \beta}^{\mu}=\frac{1}{2} g^{\mu \lambda}\left(\partial_{\alpha} g_{\beta \lambda}+\partial_{\beta} g_{\alpha \lambda}-\partial_{\lambda} g_{\alpha \beta}\right)
$$

\subsection{Curvature of space from the velocity vector}

Parallel transport of a vector is different over different paths. For redshift observations, we are interested in the parallel transport of photons across an expanding space whose rate of expansion changes with time and distance. The standard FLRW metric is generally written as a symmetrical function (Eq. 2), with $\mu, v=0 \cdots 3$. However, as demonstrated in Section 2, a further curvature term representing the divergence of space may be added to the $R$-axis as a consequence of its expansion. This requires an additional dimension represented by $z^{\prime}=\tau \cos i \psi=\tau \cosh \psi$ on the imaginary plane, with divergent angle $\psi$ and with $\mu, v=0 \cdots 4$.

Because $\psi$ is a hyperbolic angle, this geometry allows $\psi>360^{\circ}$, in contrast to local velocities that are represented by real angles and trigonometric functions. This divergence velocity is not a physical separation velocity in static space, but an observational velocity from the expansion of space itself, and this introduces a new component $\gamma_{\psi \psi}=\tau^{2} \sinh ^{2} \psi$ to the geodesic equation (Eq. 20):

$$
\mathrm{d} s^{2}=-\mathrm{d} t^{2}+\gamma_{i j}(x) \mathrm{d} x^{i} \mathrm{~d} x^{j}+\tau^{2} \sinh ^{2} \psi \mathrm{d} \psi^{2} .
$$


The time component is $-\mathrm{d} t^{2}$, the spatial component is $a(t)^{2}\left[\mathrm{~d} r^{2}+S_{k}^{2}(r) \mathrm{d} \Omega^{2}\right]$, and the expansion component is $\tau^{2} \sinh ^{2} \psi \mathrm{d} \psi^{2}$. The corresponding metric to the geodesic, $g_{\mu v}$, is:

$$
\left[\begin{array}{ccccc}
-1 & 0 & 0 & 0 & 0 \\
0 & a(t)^{2} & 0 & 0 & 0 \\
0 & 0 & a(t)^{2} S_{k}(r)^{2} & 0 & 0 \\
0 & 0 & 0 & a(t)^{2} S_{k}(r)^{2} \sin ^{2} \theta & 0 \\
0 & 0 & 0 & 0 & \tau^{2} \sinh ^{2} \psi
\end{array}\right]
$$

\subsection{Christoffel symbols and Ricci curvature}

This new curvature term introduces an extra component to Eqs. 14 and 16, with $\mathrm{d} U^{\psi} / \mathrm{d} s$ the time rate of change of the curvature of expansion. The new non-zero Christoffel symbols from Eq. (20) are then given by:

$$
\Gamma_{\psi \psi}^{t}=\tau \dot{\tau} \sinh ^{2} \psi ; \Gamma_{t \psi}^{\psi}=\Gamma_{\psi t}^{\psi}=\dot{\tau} / \tau ; \Gamma_{\psi \psi}^{\psi}=1 / \tanh \psi .
$$

The non-zero components of the Ricci tensor are now:

$$
\begin{gathered}
R_{00}=-3 \frac{\ddot{a}}{a} \\
R_{i j}=\left[\frac{\ddot{a}}{a}+2\left(\frac{\dot{a}}{a}\right)^{2}+2 \frac{K}{a^{2}}+\frac{\dot{a}}{a} \frac{\dot{\tau}}{\tau}\right] g_{i j} \\
R_{\psi \psi}=3\left(\frac{\dot{a}}{a}\right) \tau \dot{\tau} \sinh ^{2} \psi
\end{gathered}
$$

and the Ricci curvature is:

$$
R=6\left[\frac{\ddot{a}}{a}+\left(\frac{\dot{a}}{a}\right)^{2}+\frac{K}{a^{2}}+\frac{\dot{a}}{a} \frac{\dot{\tau}}{\tau}\right] .
$$

A consequence of these new non-zero Christoffel symbols (Eq. 22) is discussed in Section 8.

\subsection{The Einstein equation and mass-density tensor}

The Einstein field equation is a geometric theory of gravitation that describes gravity as a manifestation of the curvature of spacetime. In particular, the curvature of spacetime is directly related to the energy-stress tensor through the Einstein field equation (Eq. 27):

$$
R_{\mu v}-\frac{1}{2} R g_{\mu \nu}=\frac{8 \pi G}{c^{4}} T_{\mu v}-\frac{\Lambda}{c^{2}} g_{\mu \nu},
$$

where $R_{\mu v}$ and $R$ are functions of $g_{\mu v}$ and its first two derivatives, and $T_{\mu v}$ and $\Lambda$ are the stress-energy tensor and the cosmological expansion parameter respectively [18]. It may be noted that in the standard solution, the source of curvature is attributed entirely to matter, including dark matter and the mass equivalent of dark energy. Here, we are introducing an additional curvature term that directly corresponds to the expansion of the Universe.

For an ideal fluid with mass/unit volume $\rho$ and pressure $P$, the stress-energy tensor in the rest frame of the fluid is $T^{\mu}{ }_{v}=(\rho+P) U^{\mu} U_{v}+P \delta_{v}^{\mu}$, or:

$$
T_{\mu v}=(\rho+P) U_{\mu} U_{v}+P g_{\mu \nu},
$$

from which, by assuming symmetry with all off-diagonal components $=0$, setting $c=1$, and using $\mathrm{d} a / \mathrm{d} \tau=a / \tau$ (Fig. 4) and $\tau^{2}=-t^{2}$, we may solve Eq. (27) in terms of $\dot{a} / a$ and $\ddot{a} / a$.

$$
\left(\frac{\dot{a}}{a}\right)^{2}+\frac{K}{a^{2}}-\frac{1}{t^{2}}=\frac{8}{3} \pi G \rho+\frac{\Lambda}{3}
$$




$$
\left(\frac{\dot{a}}{a}\right)^{2}+2\left(\frac{\ddot{a}}{a}\right)+\frac{K}{a^{2}}-\frac{2}{t^{2}}=-8 \pi G P+\Lambda .
$$

or eliminating $\dot{a} / a$ from Eqs. 29 and 30,

$$
\begin{aligned}
& H(t)^{2}=\frac{8}{3} \pi G \rho-\frac{K}{a^{2}}+\frac{1}{t^{2}}+\frac{\Lambda}{3} \\
& \frac{\ddot{a}}{a}=-\frac{4 \pi G}{3}(\rho+3 P)+\frac{1}{2 t^{2}}+\frac{\Lambda}{3} .
\end{aligned}
$$

Defining $\rho_{c} \equiv 3 H_{0}^{2} / 8 \pi G$ as the critical density of the Universe, and setting Eq. (31) to the present epoch with $H(t)=H_{0}, a_{0}=1$, and $t=T_{0}$,

$$
\begin{gathered}
\rho_{c}=\rho_{0}-\frac{3 K_{0}}{8 \pi G}+\frac{3}{8 \pi G T_{0}^{2}}+\frac{\Lambda_{0}}{8 \pi G}, \\
\text { and defining: } \Omega_{m} \equiv \frac{8 \pi G \rho_{0}}{3 H_{0}^{2}} \quad \Omega_{K} \equiv-\frac{K}{H_{0}^{2}} \\
\Omega_{C} \equiv \frac{1}{H_{0}^{2} T_{0}^{2}} \quad \Omega_{\Lambda} \equiv \frac{\Lambda}{3 H_{0}^{2}},
\end{gathered}
$$

Eq. (33) may now be rewritten as $1=\Omega_{m}+\Omega_{K}+\Omega_{c}+\Omega_{\Lambda}$. Using $a / a_{0}=1 /(1+z), \dot{a} / a=$ $-\dot{z} /(1+z), \rho=\rho_{0}\left(a_{0} / a\right)^{3}$, and the defined density parameters, we may write [13]:

$$
d_{C}=\int_{t_{0}}^{t_{e}} \frac{d t}{a(t)}=\int_{0}^{z}\left(\frac{a}{\dot{a}}\right) d z=\int_{0}^{z} \frac{d z}{H_{0} E(z)}
$$

where $d_{C}$ is the comoving distance, $\dot{a} / a=H_{0} E(z)$, and

$$
E(z)=\left[\Omega_{m}(1+z)^{3}+\Omega_{K}(1+z)^{2}+\Omega_{C}(1+z)^{2}+\Omega_{\Lambda}\right]^{1 / 2} .
$$

\subsection{Solutions}

Letting $\Omega_{\Lambda}=\Omega_{P}=0$, and assuming a flat Euclidean Universe with $\Omega_{K}=0$, we may state $\Omega_{C}=1-\Omega_{m}$. This has an analytical solution in $z$ (Eq. 36),

$$
\begin{aligned}
& d_{C}=\frac{c}{H_{0}} \frac{1}{\sqrt{1-\Omega_{m}}} \times \\
& \log \left(\frac{(1+z)\left(\left(1-0.5 \Omega_{m}\right)+\sqrt{1-\Omega_{m}}\right)}{1+0.5 \Omega_{m}(z-1)+\sqrt{\left(1-\Omega_{m}\right)\left(1+\Omega_{m} z\right)}}\right)
\end{aligned}
$$

which reduces to $d_{C}=\left(c / H_{0}\right) \ln (1+z)$ in the Milne limit $\Omega_{m} \rightarrow 0$. This new derivation for $d_{C}$ is compared with luminosity distance measures (Section 6 ) and the recently extended angular diameter distance measures (Section 7).

\section{Luminosity Distance}

Correlation between the distance modulii derived from the standard $\Lambda \mathrm{CDM}$ and modified GR model was assessed using the extensive type Ia supernovae (SNe Ia) observations [19]. These include SN 1a data for 740 sources [19, Table F.3] covering the redshift range $0.01 \leq z \leq 1.3$ and include data from: the Supernova Legacy Survey (SNLS) [20]; the SDSS SNe survey [21]; the compilation 


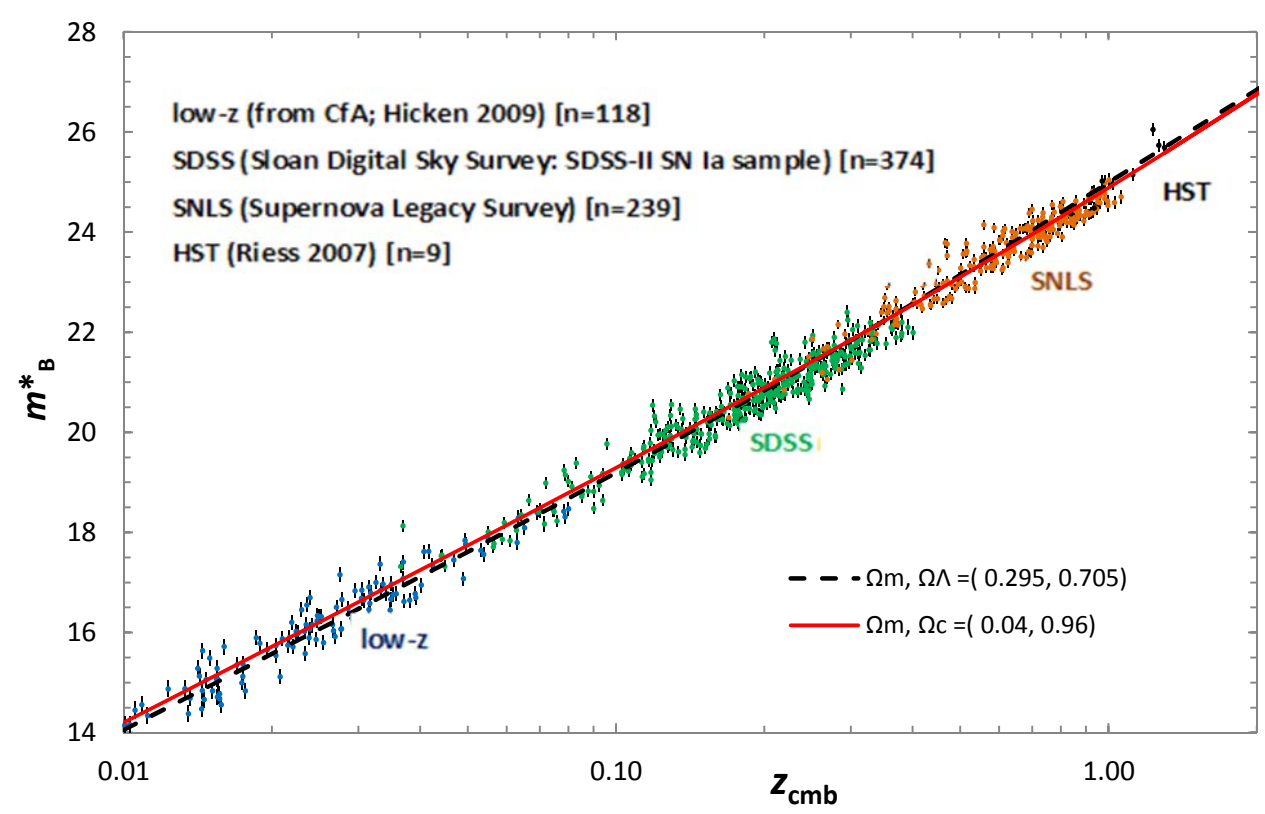

Figure 5. Hubble diagram of the combined sample of 920 SNe Ia with the observed peak magnitudes in rest frame $B$-band $\left(m_{B}^{*}\right)$ [19]. Overlain are the weighted RMS-minimisation fit for the modified GR model (solid line) and the best-fit $\Lambda \mathrm{CDM}$ cosmology with $H_{0}=70 \mathrm{~km} \mathrm{~s}^{-1} \mathrm{Mpc}^{-1}$ and $\Omega_{m}=0.295$, $\Omega_{\Lambda}=0.705$ (dashed line). Redshifts are corrected to CMB background.

comprising SNe from SNLS, HST and several nearby experiments [22]; photometry of 14 very high redshift $(0.7<z<1.3)$ SNe Ia from space-based observations with the HST [23]; and low-z $(z<$ 0.08) SNe from the photometric data acquired by the Harvard-Smithsonian Center for Astrophysics (CfA3) [24]. The corrected apparent brightness parameter $m_{B}^{*}$ for each SN Ia was plotted against its CMB-corrected redshift $\left(z_{C M B}\right)$ to create the Hubble diagram of Fig. 5 . Normalisation depends on the assumed absolute magnitude of the $\mathrm{SNe}$ and $\mathrm{H}_{0}$; varying either is equivalent to sliding the curves vertically.

Betoule et al [19] fitted a $\Lambda \mathrm{CDM}$ cosmology to the $\mathrm{SNe}$ measurements by assuming an unperturbed FLRW geometry [25], using a fixed fiducial value of $H_{0}=70 \mathrm{~km} \mathrm{~s}^{-1} \mathrm{Mpc}^{-1}\left(M_{B}=\right.$ $-19.12 \pm 0.05$ ) to obtain a best fit value for $\Omega_{m}$ of $0.295 \pm 0.034$, with $\Omega_{\Lambda}=0.705$ (dashed line). The modified GR model curve (solid line) was fitted by weighted RMS-minimisation to the full data set assuming $\Omega_{m}=0.04$ as the best current assessment of the mean total observed baryonic density of the Universe, and is comparable to that for the $\Lambda$ CDM model (weighted RMS \pm 0.016 and \pm 0.017 respectively). Their $\Lambda \mathrm{CDM}$ model is 0.15 mag fainter than the modified GR model at $z_{c m b}=1.0$, and the two curves differ by ${ }_{-0.15}^{+0.11} m_{B}^{*}$ mag over the range $0.01<z<1.3$.

Betoule et al [19] made a substantial effort to correct the distance modulus for each individual SN, using a parameter $\left(X_{1}\right)$ for time stretching of the light-curve, and a colour-correction parameter $(C)$ for the supernova colour at maximum brightness [26]. Using a corrected distance modulus $\mu_{B}=m_{B}^{*}-$ $\left(M_{B}^{*}-\alpha X_{1}+\beta C\right)$, the resultant plots had less scatter than the raw $m_{B}^{*}$ data and became progressively fainter than the $\Lambda \mathrm{CDM}$ curve with increasing $z_{c m b}$ (Fig. 6). To correct for this, they considered three alternatives to the basic $\Lambda$ CDM model: (a) a non-zero spatial curvature, $\Omega_{k} ;(b)$ a $w C D M$ model with an arbitrary constant equation of state for the dark energy with the parameter $w$ equivalent to the jerk parameter of Riess et al [27]; (c) a time-dependent equation of state with a third-order term equivalent to the snap parameter, $w^{\prime}$ [27]. They concluded that the best overall fit was to a flat universe with typical $\Omega_{k} \simeq 0.002 \pm 0.003$, and a $w \mathrm{CDM}$ model, with $w=-1.018 \pm 0.057$ (stat+sys), and with these corrections their $w \mathrm{CDM}$ curve overlays the binned plots at the faint end (Fig. 6). The modified GR 


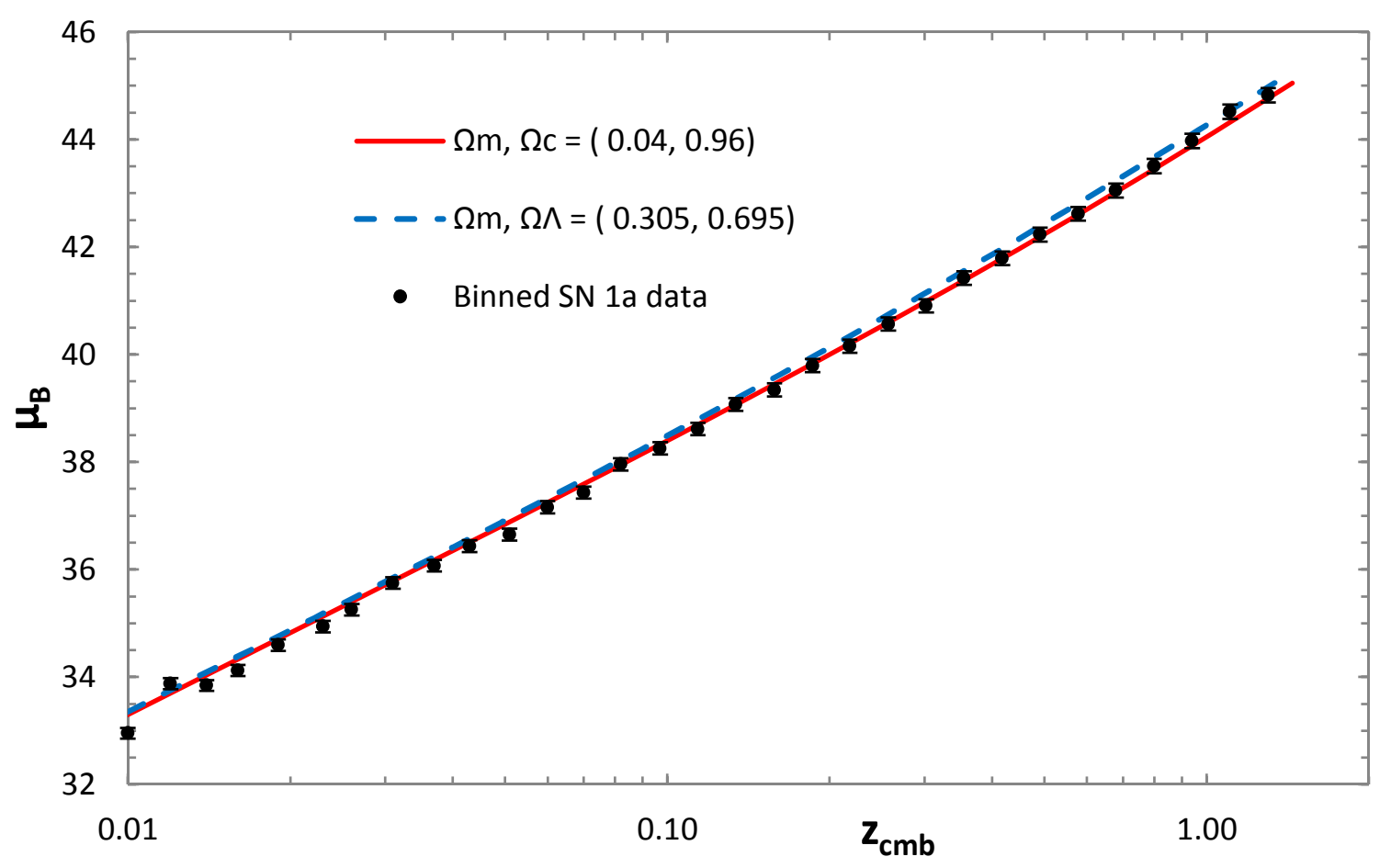

Figure 6. Hubble diagram of $920 \mathrm{SNe}$ Ia binned logarithmically in $z_{c m b}$, with corrected distance modulii $\mu_{B}$. Overlain are the unweighted least-squares fit for the modified GR model (solid line; RMS error $\pm 0.136 \mu_{B}$ mag) and the best-fit $w$ CDM cosmology with $\Omega_{m}=0.305, \Omega_{\Lambda}=0.695$ (dashed line; RMS error $\pm 0.151 \mu_{B}$ mag). Data from Betoule et al. [19] Table F.1.

model was normalised to the standard model at $z=0.01$. The overall unweighted RMS errors remain comparable for the $w \mathrm{CDM}$ and modified GR models, being \pm 0.151 and $\pm 0.136 \mu_{B}$ mag. respectively, differing by ${ }_{-0.24}^{+0.00} \mu_{B}$ mag. over the range $z_{c m b}=0.01-1.3$.

\section{Angular Diameter Distance}

\subsection{Baryon acoustic oscillations}

Angular diameter distance $d_{A}$ is defined for an object of known proper size $D$, that subtends an angle $\phi$ to the observer such that

$$
d_{A}=D / \phi .
$$

If a suitable measuring rod can be found that is independent of galactic evolution, then the points of $D$ are fixed in space and lie on the surface of the space-like sphere defined by the proper radius $\Re_{e}$ of Figs. 2 and 3, where we may identify $\Re_{e}$ with the angular size distance. This may be used with the standard expression for $d_{A}$ [37], in terms of $d_{C}$ from equation (34):

$$
d_{A}=\frac{d_{C}}{(1+z)} .
$$

Experimental verification for curves of this type is notoriously difficult because of the unknown evolution of galaxies, clusters and quasars [37-40], but recent work using the phenomenon of baryonic acoustic oscillation (BAO) has enabled measurements of $d_{A}$ with considerable accuracy.

The BAO signal is one of the key modern methods for measuring the expansion history. The BAO arose because the coupling of baryons and photons in the early Universe allowed acoustic oscillations 
Table 1. Parameters from the BAO surveys

\begin{tabular}{lccc}
\hline Survey & $z$ & $\begin{array}{c}D_{V}\left(r_{d} / r_{d, f i d}\right) \\
(\mathrm{Mpc})\end{array}$ & Ref \\
\hline 6dFGS & 0.106 & $456 \pm 27$ & 1 \\
MGS & 0.15 & $664 \pm 25$ & 2 \\
BOSS (B1) & 0.275 & $1104 \pm 30$ & 3 \\
BOSS LowZ & 0.32 & $1264 \pm 25$ & 4 \\
BOSS (B2) & 0.35 & $1356 \pm 25$ & 5 \\
WiggleZ (W1) & 0.44 & $1716 \pm 83$ & 6 \\
CMASS & 0.57 & $2056 \pm 20$ & 4 \\
WiggleZ (W2) & 0.6 & $2221 \pm 101$ & 6 \\
WiggleZ & 0.73 & $2516 \pm 86$ & 6 \\
Lyman- $\alpha$ forest & 2.36 & $6474 \pm 163$ & 7 \\
\hline
\end{tabular}

(1) Beutler et al. [28]; (2) Ross et al. [29]; (3) Percival et al. [30]; (4) Anderson et al. [31], Cuesta et al. [32]; (5) Padmanabhan et al. [33], Xu et al. [34]; (6) Kazin et al. [35]; (7) Font-Ribera et al. [36]

to develop that led to anisotropies of the cosmic microwave background (CMB) radiation and a rich structure in the distribution of matter [41,42]. The acoustic scale length $\left(r_{S}\right)$ can be computed as the comoving distance that the sound waves could travel from the Big Bang until recombination. The imprint left by these sound waves provides a feature of known size in the late-time clustering of matter and galaxies, and by measuring this acoustic scale at a variety of redshifts, one can infer $d_{A}(z)$ and $H(z)$.

Determination of $r_{S}$ comes from the the matter-to-radiation ratio and the baryon-to-photon ratio, both of which are well measured by the relative heights of the acoustic peaks in the CMB anisotropy power spectrum [43,44]. Both cosmological perturbation theory and numerical simulations suggest that this feature is stable to better than $1 \%$ accuracy, making it an excellent standard ruler. The propagation distance of the acoustic waves becomes a characteristic comoving scale fixed by the recombination time of the Universe after approximately 379,000 years, at a redshift of $z \simeq 1089$ [45-47]. Eisenstein et al provide a discussion of the acoustic signal in configuration space [48], and reviews of $\mathrm{BAO}$ as a probe of dark energy [49]. The acoustic scale is expressed in absolute units (Mpc) rather than $h^{-1} \mathrm{Mpc}$, and is imprinted on very large scales $(\sim 150 \mathrm{Mpc})$ thereby being relatively insensitive to small scale astrophysical processes, making BAO experiments less sensitive to this type of systematic error [44].

Figure 7 combines the $\mathrm{BAO}$ results from a number of sources using spectroscopic data sets, and the quasar Lyman- $\alpha$ results from the SDSS-III Baryon Oscillation Spectroscopic Survey (BOSS). The volume $D_{V}(z)$ corresponds to the peak position for an isotropic distribution of galaxy pairs and the 2-point isotropic clustering strength $\xi(z)$ of the observations, computed using Eq. (39) to convert the line-of-sight distance into an equivalent transverse length scale, where $d_{A}$ is the angular diameter distance and $H(z)$ is the Hubble parameter in the appropriate model. As the BAO method actually measures $D_{V} / r_{d}$, this quantity was multiplied by the fiducial scale length $r_{s, \text { fid }}$ to restore a distance $[42,51]$.

$$
D_{V} \equiv\left[d_{A}^{2} \times \frac{c z}{H(z)}(1+z)^{2}\right]^{1 / 3}
$$

Included are the acoustic peak detection from the 6dF Galaxy Survey at $z=0.106$ [28]; the MGS survey at $z=0.15$ [29]; a combination of Sloan Digital Sky Survey (SDSS)-II DR7 LRG and main sample galaxies combined with the $2 \mathrm{dF}$ data (B1) at $z=0.275$ [30]; the BOSS CMASS measurements at $z=0.32$ and $z=0.57$ [31,32]; the SDSS-II LRG (B2) measurement at $z=0.35$ using reconstruction to sharpen the precision of the BAO measurement $[33,34]$; and the WiggleZ measurement of three 


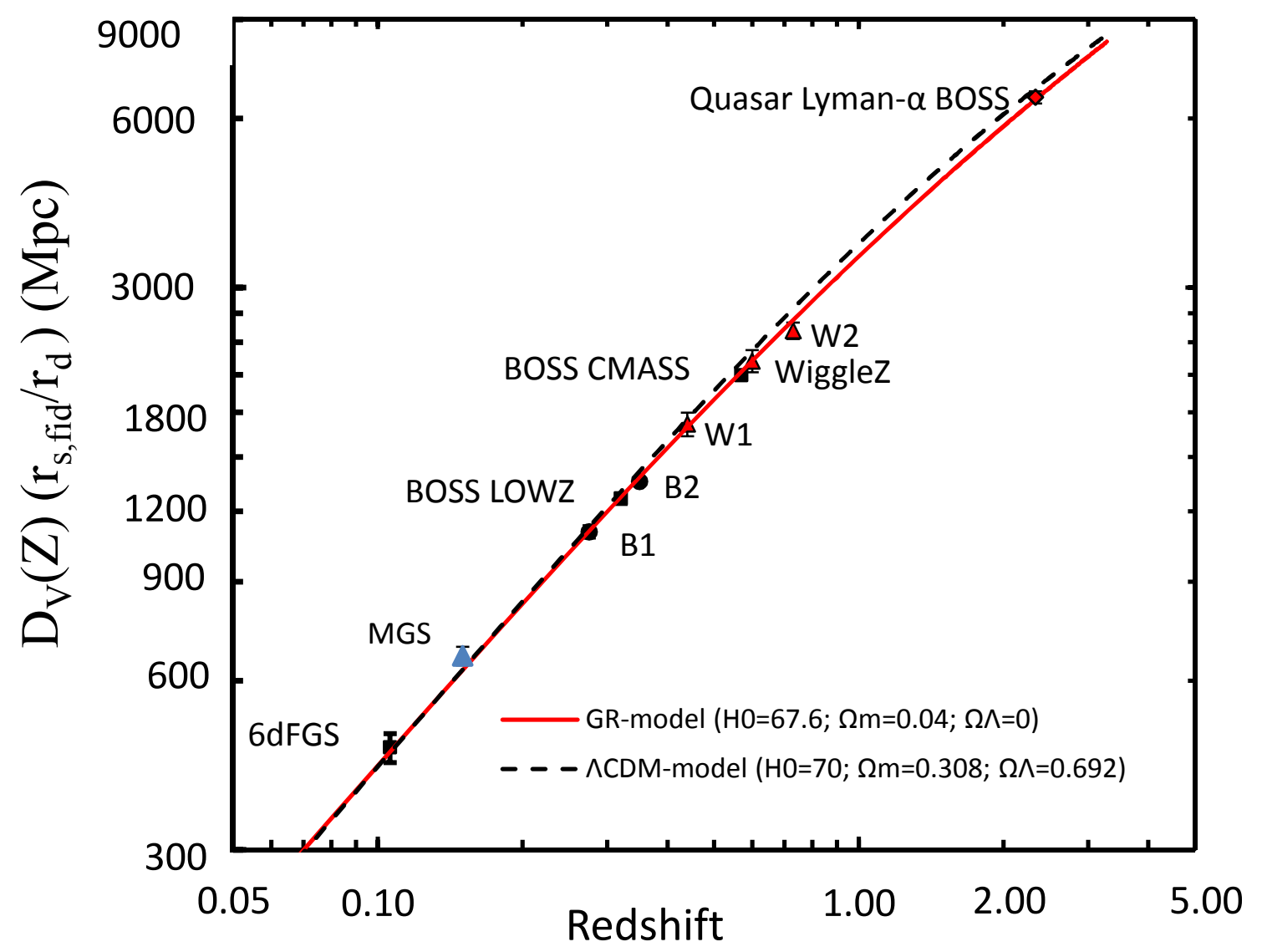

Figure 7. A plot of the distance-redshift relation from the spectroscopic data BAO measurements and quasar Lyman- $\alpha$ BOSS, plotting $D_{V}(z)\left(r_{s, f i d} / r_{d}\right)$ (Table 1). Overlain are the modified GR model fitted by weighted RMS-minimisation to $H_{0}=67.6 \pm 0.25$ with $\Omega_{m}=0.04, \Omega_{C}=0.96$ (red solid line) and the best-fitting flat $\Lambda$ CDM 1- $\sigma$ prediction from WMAP under the assumption of a flat universe with a cosmological constant $\left(\Omega_{m}=0.308 ; \Omega_{\Lambda}=0.692\right)[42,50]$ (dashed line).

partially covariant data sets at $z=0.44,0.6$, and 0.73 [35]. The published values for $D_{V}(z)$ are presented in Table 1. Font-Ribera et al [36] measured the large-scale cross-correlation of quasars with the Lyman- $\alpha$ forest absorption, using over 164,000 quasars from DR11 of the SDSS-III BOSS. Their result was an absolute measure of $d_{A}=1590 \pm 60 \mathrm{Mpc}$ at $z=2.36$, equivalent to $D_{V}=6474 \pm$ $163\left(r_{d} / r_{s, f i d}\right) \mathrm{Mpc}$, with $r_{d}=147.49 \mathrm{Mpc}$.

The data of Fig. 7 are overlain with the best-fit curves for the two models. The solid curve is the modified GR model with $\Omega_{m}=0.04, \Omega_{C}=0.96$, and the dashed line is the $\Lambda$ CDM prediction from WMAP under the assumption of a flat universe with a cosmological constant using Planck Collaboration data $\left(\Omega_{m}=0.308 \pm 0.012 ; \Omega_{\Lambda}=0.692 \pm 0.012 ; \Omega_{K}=0\right)$ [7].

As in Section 6, changing $H_{0}$ slides the curves up or down the vertical axis, but does not alter the shapes of the curves which were fitted by weighted RMS minimisation against the combined BAO samples of Table 1 to give $H_{0}=67.6 \pm 0.25$ with weighted RMS errors of $\pm 0.034 \mathrm{Mpc}$ for the modified GR model, in good concordance with the most recent Planck results of $H_{0}=67.4 \pm 0.5$ [7], rather than the high values suggested by the distance scale $\left(H_{0}=73.24 \pm 1.7\right)$ [52]. The uncertainties in the former come from $\Omega_{m} h^{2}$, and uncertainties in the later largely from Gaia parallax distances of Cepheid calibrators. For the $\Lambda$ CDM model, $H_{0}=70.0 \pm 0.25$ with weighted RMS errors \pm 0.085 Mpc which is intermediate between the two extremes, but the standard model can be improved with non-linear parameters added to $\Omega_{\Lambda}$ in a $w^{\prime}$-CDM model. 


\section{Discussion}

By considering GR as a geometrical manifold with an imaginary time-axis, adjacent photon paths trace out a thin ribbon that everywhere subtends an angle of $45^{\circ}$ to the expanding time axes, this being the locally Minkowskian metric. In a static universe with no relative velocity between emitter and receiver, this is a plane ribbon-like Euclidean quadrilateral with parallel photon paths and time-lines, and it retains this form when wrapped round a cylinder. In the Milne SR model this ribbon becomes curved and stretched by the relative velocity of emitter and receiver causing an intrinsic curvature of space to produce the observed redshift. This curvature, however, can still be wrapped round a uniform cone (Figs. 1, 2).

In contrast, the presence of mass-energy represented by $\rho_{0}$ and $P$ generates an additional curvature and twist in the ribbon (Figs. 3 and 4) that require Einstein's equations and is generally solved using the standard FLRW metric. Assuming spatial curvature to be zero, the observed matter in the Universe is insufficient to account for the measured redshifts and requires the inclusion of an additional dark-matter component, while to conform to the more detailed SNe 1a measurements an additional dark-energy $\Lambda$ acceleration term is included, mathematically equivalent to a gravitationally repulsive negative mass [53]. Deeper and more detailed SNe 1a measurements $[19,27]$ have required second and third order refinements to $\Lambda$, with jerk $(w)$ and snap $\left(w^{\prime}\right)$ parameters.

While the nature of dark matter and dark energy remain elusive [54], several alternative theories to standard GR have emerged such as scale invariance as an alternative to dark energy [55]. However, recently published work following the observation of gravitational waves from the binary neutron star GW170817 [56] have determined $c_{g}$ with sufficient accuracy to suggest that $c_{g}=c \pm 10^{-15}$. This has eliminated some alternative scenarios proposed to account for the unobserved dark energy fraction of Einstein's equation $[57,58]$ and several gravitational theories that predict an anomalous $c_{g}$ propagation speed such as some MOND-like gravities including Tensor-vector-scalar gravity (TeVeS), Hernández forms, Einstein-Aether, Generalised Proca, and Hořava gravity [59].

A central tenet of GR is that it is always valid to choose a coordinate system that is locally Minkowskian. This was developed further by Chodorowski [60] who suggested that the frequency shift coincides with decomposition into a Doppler (kinematic) component and a gravitational one, and by Kaiser [61] who suggested that even where gravitational redshift dominates, redshift can always be formally expressed using the Doppler formula such that the observed cosmological redshift can be interpreted as either a gravitational redshift, or a kinematic redshift by the integration of infinitesimal Doppler shifts. Performing such a transport along the null geodesic of photons arriving from a receding galaxy, they considered that the frequency shift is purely kinematic, corresponding to a family of comoving observers, and hence was more natural.

The extension to GR presented in this paper incorporates both kinematic and gravitational components as $\Omega_{C}$ and $\Omega_{m}$ respectively, with parallel transport along the photon path, and rotation across curved diverging time lines. Non-zero Christoffel symbols are imposed by any acceleration, whether caused by a gravitational field, by the action of fields other than those associated with gravitational mass, or by curvilinear motion [62]. The emergence of new non-zero Christoffel symbols (Eq. 22) supports the presence of curvilinear motion imposed on the red-shifted photons by the expansion of space that is distinct from the curvature of space by the presence of mass. The two curvature terms, $\Omega_{K}$ and $\Omega_{C}$, are derived from quite different principles, the former being an intrinsic curvature within space itself, while the latter emerges from the Hubble flow.

The introduction of an additional curvature term into Einstein's equation follows directly from the geometry of Hubble expansion and is a logical extension to the standard model in expanding space [9]. The proposed modified GR model can accommodate a scenario in which the observations of $\mathrm{SNe}$ 1a and BAO do not require additional parameters from DM or accelerating dark energy. The use of $\Omega_{C}$ as a Hubble curvature allows a smooth transition to the Einstein equation for full GR as density increases from zero, without requiring a discontinuity in the curvature parameter, $\Omega_{K}$. The introduction of $\Omega_{C}$ generates a magnitude-redshift curve that well matches current $\mathrm{SNe}$ 
1a observations out to $z=1.3$, assuming only that $\rho_{m}$ represents observable baryonic mass. BAO measurements for angular diameter distances also give an excellent fit from low- $z$ out to $z=2.36$, without including additional or arbitrary parameters. Weighted RMS-minimisation fitting to the combined BAO samples of Table 1 gave $H_{0}=67.6 \pm 0.25$, in good concordance with the recent Planck results [7]. DM may still be required within galaxies to account for galactic rotation, gravitational lensing, and the motion of clusters, but the inclusion of a Hubble expansion curvature term in GR can account for many recent cosmological observations without requiring cosmological DM or dark energy terms.

\section{Acknowledgments}

My thanks to Professor Tom Shanks and Dr Nigel Metcalfe of Durham University for their encouragement and insights into computational methods and observational geometry.

Conflicts of Interest: The author declares no conflict of interest.

\section{Bibliography}

1. Cottam, J.; Paerels, F.; Mendez, M. Gravitationally Redshifted Absorption Lines in the Burst Spectra of the Neutron Star in EXO 0748-676. High Resolution X-ray Spectroscopy with XMM-Newton and Chandra; Branduardi-Raymont, G., Ed., 2002.

2. Wong, K.C.; Suyu, S.H.; Matsushita, S. The Innermost Mass Distribution of the Gravitational Lens SDP.81 from ALMA Observations. ApJ 2015, 811, 115, [1503.05558].

3. Müller, A.; Camenzind, M. Relativistic emission lines from accreting black holes. The effect of disk truncation on line profiles. A\&Ap 2004, 413, 861-878, [astro-ph/0309832].

4. Bromley, B.C.; Kenyon, S.J.; Brown, W.R.; Geller, M.J. Runaway Stars, Hypervelocity Stars, and Radial Velocity Surveys. ApJ 2009, 706, 925-940, [0907.5567].

5. Reynolds, M.T.; Reis, R.C.; Miller, J.M.; Cackett, E.M.; Degenaar, N. The quiescent X-ray spectrum of accreting black holes. MNRAS 2014, 441, 3656-3665, [arXiv:astro-ph.HE/1405.0474].

6. Crowell, B. General Relativity; Fullerton: California, 2012.

7. Planck Collaboration. Aghanim, N.; Akrami, Y..; Ashdown, M..; Aumont, J.; Baccigalupi, C..; Ballardini, M..; et. al.. Planck 2018 results - VI. Cosmological parameters. A\&Ap 2020, 641, A6.

8. D'Amico, G.; Senatore, L.; Zhang, P. Limits on wCDM from the EFTofLSS with the PyBird code. J. Cosmology Astropart. Phys. 2021, 2021, 006, [arXiv:astro-ph.CO/2003.07956].

9. Marr, J.H. A novel visualization of the geometry of special relativity. Int. J. Mod. Phys. C 2016, 27, 1650055-302, [arXiv:physics.class-ph/1511.02197].

10. Milnor, J. Towards the Poincare Conjecture and the Classification of 3-Manifolds. NOTICES OF THE AMS 2003, 50, $1226-1233$.

11. Perelman, G. Ricci flow with surgery on three-manifolds. arXiv:math/0303109v1 2008.

12. Misner, C.W.; Thorne, K.S.; Wheeler, J.A. Gravitation; W. H. Freeman and Company: San Francisco, 1970.

13. Peebles, P.J.E. Principles of Physical Cosmology; Princeton University Press: Princeton NJ, 1993.

14. Carroll, S.M. An Introduction to General Relativity Spacetime and Geometry; Addison-Wesley: San Francisco, 2003.

15. Komissarov, S.S.; Hughes, P.A.; Bregman, J.N. On Some Recent Developments in Numerical Methods for Relativistic MHD. AIP Conference Proceedings 2006, 856, 129-149.

16. Figueras, P.; Kunesch, M.; Tunyasuvunakool, S. End Point of Black Ring Instabilities and the Weak Cosmic Censorship Conjecture. Phys. Rev. Lett. 2016, 116, 071102.

17. Milne, E.A. Relativity, Gravitation and World Structure; OUP: Oxford, UK, 1935.

18. Gopal Vishwakarma, R. Mysteries of the geometrization of gravitation. Research in Astronomy and Astrophysics 2013, 13, 1409-1422, [arXiv:physics.gen-ph/1206.5789].

19. Betoule, M.; Kessler, R.; Guy, J.; Mosher, J.; Hardin, D.; Biswas, R.; et al. Improved cosmological constraints from a joint analysis of the SDSS-II and SNLS supernova samples. AEAp 2014, 568, A22, [1401.4064]. 
20. Astier, P.; Guy, J.; Regnault, N.; Pain, R.; Aubourg, E.; Balam, D.; et al. The Supernova Legacy Survey: measurement of $\Omega_{M}, \Omega_{\Lambda}$ and $w$ from the first year data set. AEAp 2006, 447, 31-48, [astro-ph/0510447].

21. Sako, M.; Bassett, B.; Becker, A.C.; Brown, P.J.; Campbell, H.; Cane, R.; et al. The Data Release of the Sloan Digital Sky Survey-II Supernova Survey. PASP 2014, [arXiv:astro-ph.CO/1401.3317].

22. Conley, A.; Guy, J.; Sullivan, M.; Regnault, N.; Astier, P.; Balland, C.; et al. Supernova Constraints and Systematic Uncertainties from the First Three Years of the Supernova Legacy Survey. ApJS 2011, 192, 1, [arXiv:astro-ph.CO/1104.1443].

23. Riess, A.G.; Strolger, L.G.; Casertano, S.; Ferguson, H.C.; Mobasher, B.; Gold, B.; et al. New Hubble Space Telescope Discoveries of Type Ia Supernovae at $\mathrm{z} \geq 1$ : Narrowing Constraints on the Early Behavior of Dark Energy. ApJ 2007, 659, 98-121, [astro-ph/0611572].

24. Hicken, M.; Challis, P.; Jha, S.; Kirshner, R.P.; Matheson, T.; Modjaz, M.; et al. CfA3: 185 Type Ia Supernova Light Curves from the CfA. ApJ 2009, 700, 331-357, [arXiv:astro-ph.CO/0901.4787].

25. Sandage, A. Observational tests of world models. ARAEA 1988, 26, 561-630.

26. Tripp, R. A two-parameter luminosity correction for Type IA supernovae. AEAp 1998, 331, 815-820.

27. Riess, A.G.; Strolger, L.G.; Tonry, J.; Casertano, S.; Ferguson, H.C.; Mobasher, B.; et al. Type Ia Supernova Discoveries at z geq 1 from the Hubble Space Telescope: Evidence for Past Deceleration and Constraints on Dark Energy Evolution. ApJ 2004, 607, 665-687, [astro-ph/0402512].

28. Beutler, F.; Blake, C.; Colless, M.; Jones, D.H.; Staveley-Smith, L.; Campbell, L.; et al. The 6dF Galaxy Survey: baryon acoustic oscillations and the local Hubble constant. MNRAS 2011, 416, 3017-3032, [1106.3366].

29. Ross, A.J.; Samushia, L.; Howlett, C.; Percival, W.J.; Burden, A.; Manera, M. The clustering of the SDSS DR7 main Galaxy sample - I. A 4 per cent distance measure at $\mathrm{z}=0.15$. MNRAS 2015, 449, 835-847, [1409.3242].

30. Percival, W.J.; Reid, B.A.; Eisenstein, D.J.; Bahcall, N.A.; Budavari, T.; Frieman, J.A.; et al. Baryon acoustic oscillations in the Sloan Digital Sky Survey Data Release 7 galaxy sample. MNRAS 2010, 401, 2148-2168, [arXiv:astro-ph.CO/0907.1660].

31. Anderson, L.; Aubourg, É.; Bailey, S.; Beutler, F.; Bhardwaj, V.; Blanton, M.; et al. The clustering of galaxies in the SDSS-III Baryon Oscillation Spectroscopic Survey: baryon acoustic oscillations in the Data Releases 10 and 11 Galaxy samples. MNRAS 2014, 441, 24-62, [1312.4877].

32. Cuesta, A.J.; Vargas-Magaña, M.; Beutler, F.; Bolton, A.S.; Brownstein, J.R.; Eisenstein, D.J.; et al. The clustering of galaxies in the SDSS-III Baryon Oscillation Spectroscopic Survey: baryon acoustic oscillations in the correlation function of LOWZ and CMASS galaxies in Data Release 12. MNRAS 2016, 457, 1770-1785, [1509.06371].

33. Padmanabhan, N.; Xu, X.; Eisenstein, D.J.; Scalzo, R.; Cuesta, A.J.; Mehta, K.T.; Kazin, E. A 2 per cent distance to $\mathrm{z}=0.35$ by reconstructing baryon acoustic oscillations $-\mathrm{I}$. Methods and application to the Sloan Digital Sky Survey. MNRAS 2012, 427, 2132-2145, [1202.0090].

34. Xu, X.; Cuesta, A.J.; Padmanabhan, N.; Eisenstein, D.J.; McBride, C.K. Measuring $\mathrm{D}_{A}$ and $\mathrm{H}$ at $\mathrm{z}=0.35$ from the SDSS DR7 LRGs using baryon acoustic oscillations. MNRAS 2013, 431, 2834-2860, [1206.6732].

35. Kazin, E.A.; Koda, J.; Blake, C.; Padmanabhan, N.; Brough, S.; Colless, M.; et al. The WiggleZ Dark Energy Survey: improved distance measurements to $\mathrm{z}=1$ with reconstruction of the baryonic acoustic feature. MNRAS 2014, 441, 3524-3542, [1401.0358].

36. Font-Ribera, A.; Kirkby, D.; Busca, N.; Miralda-Escudé, J.; Ross, N.P.; Slosar, A.; et al. Quasar-Lyman $\alpha$ forest cross-correlation from BOSS DR11: Baryon Acoustic Oscillations. J. Cosmology Astropart. Phys. 2014, 5, 027, [1311.1767].

37. Bonamente, M.; Joy, M.K.; LaRoque, S.J.; Carlstrom, J.E.; Reese, E.D.; Dawson, K.S. Determination of the Cosmic Distance Scale from Sunyaev-Zel'dovich Effect and Chandra X-Ray Measurements of High-Redshift Galaxy Clusters. ApJ 2006, 647, 25-54, [astro-ph/0512349].

38. Hogg, D.W. Constraints on Photometric Calibration from Observations of High-Redshift Type Ia Supernovae. arXiv:astro-ph/0001419 2000.

39. Ruhl, J.; Ade, P.A.R.; Carlstrom, J.E.; Hsiao-Mei Cho, H.; et. al.. Millimeter and Submillimeter Detectors for Astronomy II. The South Pole Telescope, 2004, Vol. 5498, pp. 5498 - 5498 - 19. 
40. Bleem, L.E.; Stalder, B.; de Haan, T.; Aird, K.A.; Allen, S.W.; Applegate, D.E.; et al. Galaxy Clusters Discovered via the Sunyaev-Zel'dovich Effect in the 2500-Square-Degree SPT-SZ Survey. ApJS 2015, 216, 27, [1409.0850].

41. Glazebrook, K.; Blake, C. Measuring the Cosmic Evolution of Dark Energy with Baryonic Oscillations in the Galaxy Power Spectrum. ApJ 2005, 631, 1-20, [astro-ph/0505608].

42. Anderson, L.; Aubourg, E.; Bailey, S.; Bizyaev, D.; Blanton, M.; Bolton, A.S.; et al. The clustering of galaxies in the SDSS-III Baryon Oscillation Spectroscopic Survey: baryon acoustic oscillations in the Data Release 9 spectroscopic galaxy sample. MNRAS 2012, 427, 3435-3467, [1203.6594].

43. Eisenstein, D.J.; Hu, W.; Tegmark, M. Cosmic Complementarity: $\mathrm{H}_{0}$ and $\Omega_{m}$ from Combining Cosmic Microwave Background Experiments and Redshift Surveys. ApJL 1998, 504, L57-L60, [astro-ph/9805239].

44. Weinberg, D.H.; Mortonson, M.J.; Eisenstein, D.J.; Hirata, C.; Riess, A.G.; Rozo, E. Observational probes of cosmic acceleration. Phys. Rep. 2013, 530, 87-255, [1201.2434].

45. Peebles, P.J.E.; Yu, J.T. Primeval Adiabatic Perturbation in an Expanding Universe. ApJ 1970, $162,815$.

46. Sunyaev, R.A.; Zeldovich, Y.B. Small-Scale Fluctuations of Relic Radiation. ApESS 1970, 7, 3-19.

47. Doroshkevich, A.G.; Zel'dovich, Y.B.; Syunyaev, R.A. Fluctuations of the microwave background radiation in the adiabatic and entropic theories of galaxy formation. Soviet Ast. 1978, 22, 523-528.

48. Eisenstein, D.J.; Seo, H.J.; White, M. On the Robustness of the Acoustic Scale in the Low-Redshift Clustering of Matter. ApJ 2007, 664, 660-674, [astro-ph/0604361].

49. Eisenstein, D.J.; Bennett, C.L. Cosmic sound waves rule. Physics Today 2008, 61, 44.

50. Komatsu, E.; Smith, K.M.; Dunkley, J.; Bennett, C.L.; Gold, B.; Hinshaw, G.; et al. Seven-year Wilkinson Microwave Anisotropy Probe (WMAP) Observations: Cosmological Interpretation. ApJS 2011, 192, 18, [arXiv:astro-ph.CO/1001.4538].

51. Eisenstein, D.J.; Zehavi, I.; Hogg, D.W.; Scoccimarro, R.; Blanton, M.R.; Nichol, R.C.; et al. Detection of the Baryon Acoustic Peak in the Large-Scale Correlation Function of SDSS Luminous Red Galaxies. ApJ 2005, 633, 560-574, [astro-ph/0501171].

52. Shanks, T.; Hogarth, L.M.; Metcalfe, N. Gaia Cepheid parallaxes and 'Local Hole' relieve $\mathrm{H}_{0}$ tension. MNRAS 2019, 484, L64-L68, [arXiv:astro-ph.CO/1810.02595].

53. Perlmutter, S.; Aldering, G.; Goldhaber, G.; Knop, R.A.; Nugent, P.; Castro, P.G.; et al. Measurements of $\Omega$ and $\Lambda$ from 42 High-Redshift Supernovae. ApJ 1999, 517, 565-586, [astro-ph/9812133].

54. Slosar, A.; Mandelbaum, R.; Eisenstein, D. Dark Energy and Modified Gravity. BAAS 2019, 51, 97, [arXiv:astro-ph.CO/1903.12016].

55. Maeder, A. An Alternative to the $\Lambda$ CDM Model: The Case of Scale Invariance. ApJ 2017, 834, 194, [1701.03964].

56. The LIGO Scientific Collaboration.; the Virgo Collaboration.; Abbott, B.P.; Abbott, R.; Abbott, T.D.; Abernathy, M.R.; Acernese, F.; Ackley, K.; et al.. All-sky search for long-duration gravitational wave transients in the first Advanced LIGO observing run. Ap. J. Letters 2017, 848, L12, [arXiv:gr-qc/1711.06843].

57. Sakstein, J.; Jain, B. Implications of the Neutron Star Merger GW170817 for Cosmological Scalar-Tensor Theories. Physical Review Letters 2017, 119, 251303, [1710.05893].

58. Lombriser, L.; Lima, N.A. Challenges to self-acceleration in modified gravity from gravitational waves and large-scale structure. Physics Letters B 2017, 765, 382-385, [1602.07670].

59. María Ezquiaga, J.; Zumalacárregui, M. Dark Energy after GW170817: dead ends and the road ahead. arXiv:1710.05901 2017, [1710.05901].

60. Chodorowski, M.J. The kinematic component of the cosmological redshift. MNRAS 2011, 413, 585-594, [0911.3536].

61. Kaiser, N. Astronomical redshifts and the expansion of space. MNRAS 2014, 438, 2456-2465, [1312.1190].

62. Weinberg, S. Gravitation and Cosmology: Principles and Applications of the General Theory of Relativity; Wiley: New York, 1972. 